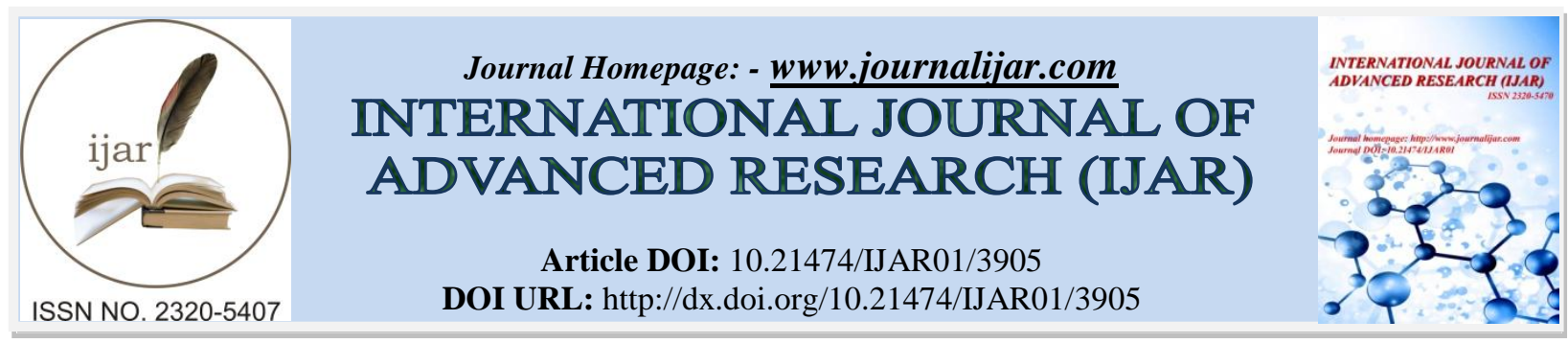

RESEARCH ARTICLE

\title{
ENDODONTIC TREATMENT OF MANDIBULAR INCISORS WITH TWO ROOT CANALS: A CASE REPORT.
}

B.G.Yogesh, M. Mahboob Ali, P.V.Sreedevi, Arun J Kumar, Ashwini B and Vatsala R.

\section{Manuscript Info}

Manuscript History

Received: 08 February 2017

Final Accepted: 05 March 2017

Published: April 2017

Key words:-

Mandibular incisor, Canal configuration,

Cone beam computed tomography.

\begin{abstract}
A wide morphological divergence of the root canal system is known to exist. Varying number of the root canals in different teeth, their anatomy and interconnections have been studied and reported.This case report describes the successful endodontic treatment of mandibular incisors having two root canals in the same individual. Radiographic examination revealed multiple canals in all mandibular central and lateral incisors.Endodontic intervention resulted in successful treatment of this unusual canal morphology. Literature reveals multiple canals in the mandibular central and lateral incisors teeth individually, but the highlight of this case report is finding of two canals in all four mandibular central and lateral incisors in same individuals.
\end{abstract}

Copy Right, IJAR, 2017,. All rights reserved.

\section{Introduction:-}

A canal is often left untreated because the dentist fails to recognize its presence either due to lack of knowledge of root canal morphology or due to lack of experience and skills to negotiate that canal. ${ }^{1}$

Sucessful endodontic therapy of a tooth demands that the dentist should have a thorough knowledge of the root canal morphology, making it mandatory towards thorough radiographic evaluation and diagnosis of the status of the pulp canals as well as the periapical areas. Improper diagnostic protocol may lead to the failure of endodontic treatment. $^{2}$

In reviewing the literatures, one finds a difference of opinion concerning the anatomic configuration of the pulp cavity and the incidence of two root canals of the mandibular incisors especially as they are difficult to access and the second canal usually appears to hide under the cingulum.

A wide morphological divergence of the root canal systems is known to exist. Varying number of the root canals in different teeth, their anatomy and interconnections have been studied and reported by several authors. Vertucci et $\mathrm{al}^{11}$ has classified morphological patterns of the root canal system into eight types. Generally, the mandibular incisors have one root canal with one apical foramen (Vertucci type II).

However the occurrence of two root canals with two separate foramina (Vertucci type IV) in the mandibular incisors is very rare viz 3\% and 2\% in the mandibular central incsors and lateral incisors respectively, and in canines it is $6 \%$.Funato $\mathrm{A}$ et al ${ }^{10}$ has reported a case with two root canals and seprate apical foamina in the mandibular central incisor. $^{4}$ 
The purpose of this case report are to illustrate the outcome of endodontic treatment of a patient who had two separate root canals in all four mandibular incisors, and to discuss the signs suggesting the presence of second canals in mandibular incisors.

\section{Case Report:-}

A 35-year-old female patient reported to the Department of Conservative dentistry and Endodontics, Farooqia dental college, Mysore (India), with a chief complaint of pain in lower anterior teeth region since 8 days. Pain was continous and dull in nature. There was no history of trauma and the medical history was non contributory. Intra oral hard tissue examination revealed deep caries in relation to 43 and fractured restoration in relation to 42.(Fig1)

Restoration was evident with respect to 32,41 and 42 which is suggestive of piror dental treatment from private clinic with no relief from symptoms. While taking dental history, patient reveal that she had visited private dental clinic for pain in 31, 32, 41 and 42 where she had undergone some dental treatment with lower anterior teeth. But, even after several visits, there was no relief from pain in 33 and 32.

On thermal and electric pulp testing both the teeth showed negative response. Radiographic examinations revealed periapical radiolucency. Diagnosis was made as chronic irreversible pulpitis w.r.t 43 \& 42. Treatment plan included root canal therapy followed by crowns. Local anaesthesia was administered. Under rubber dam isolation, access opening of 43, 42 were modified and widened buccolingually and extended into cingulum gingivally, which revelaed the presence of a lingual canal w.r.t 42. (Fig2)

The patency was checked using a no.10 k file. The working length was established using an apex locator (Root ZX Mini Morita,USA) and also confirmed radiographically to determine the anatomic relationship between the root canals of each tooth. To ensure that additional root canals were not overlooked, it was necessary to image the tooth from different angles.

After confirmation of two root canals in all lower incisors using radiovisiography (rvg) the canal was prepared using a astep back instrumentation technique. It was initially instrumented with a hand K-files (Dentsply Maillefer, Ballaigues, Switzerland) and an adequate working length was determined based on radiograph. The disinfection was carried out with 3\% sodium hypochlorite and with the use of K-files sequentially from sizes \#15 to 40 (Dentsply, Maillefer, Ballaigues Switzerland). A 3\% solution of sodium hypochlorite and normal saline were alternatively used as irrigant at every change of instruments. The access cavities were then temporarily sealed with IRM. (Fig3)

At 2 weeks follow up as the teeth were asymptomatic, obturation of the root canals was undertaken using lateral condensation technique and AH Plus sealer. Post obturation radiograph showed well obturated 2 canals in lower incisors (42) and the access cavities were sealed with IRM. (Fig 4 \& 5)
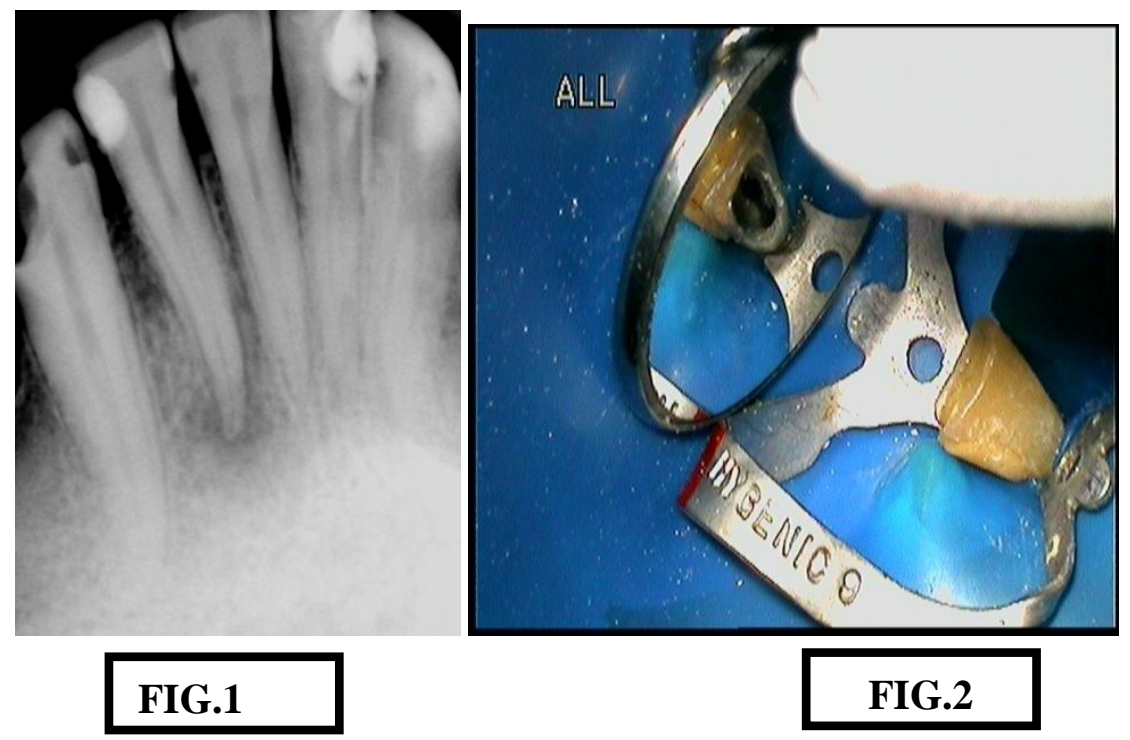

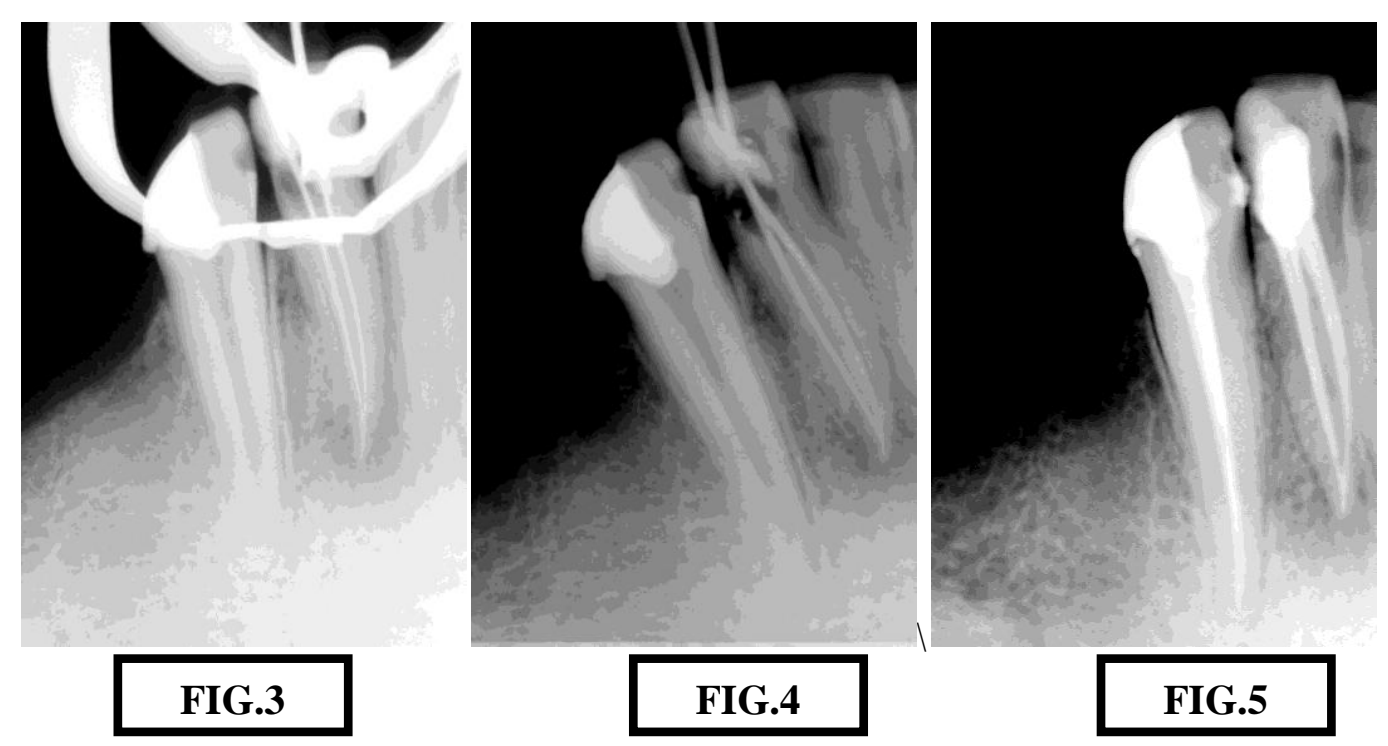

A cone beam computed tomography (CBCT) image was taken to reconfirm the root canal configuration of the remaining teeth.
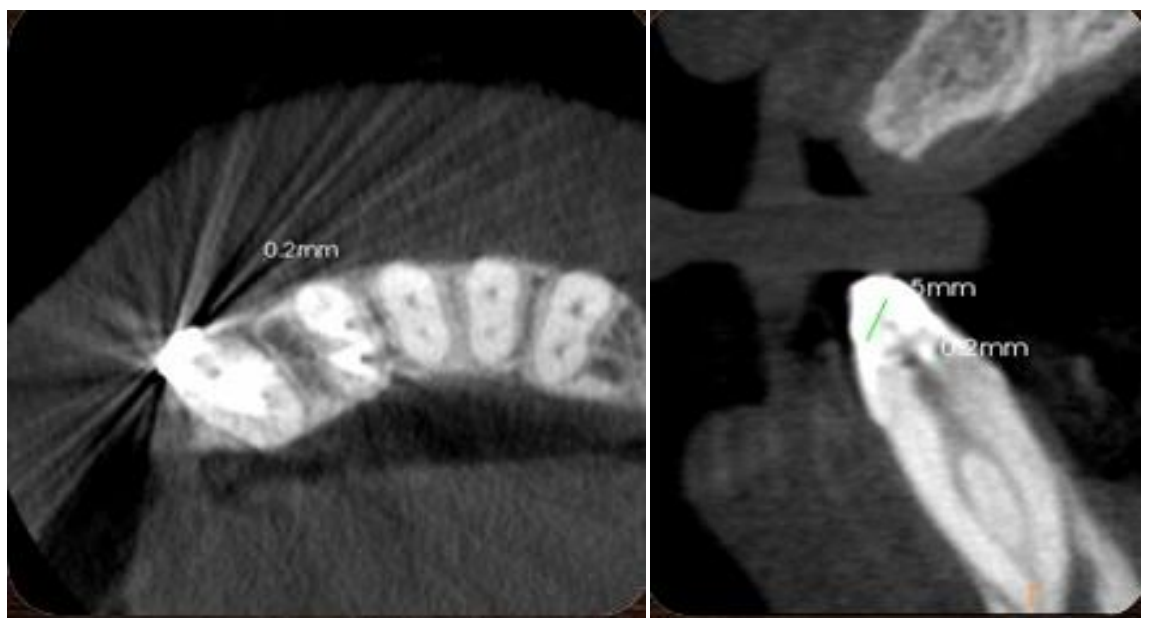

\section{Discussion:-}

A well-designed access preparation is essential for a good endodontic result. Without adequate access, instruments and materials become difficult to handle properly in the highly complex and variable root canal system. Proper access cavity preaparation provides staraight or direct line access to the apical foramina or at least to the initial curvature of canal to locate all root canal orifices and it also conserves sound tooth structure.

Since the success of the endodontic treatment is related to a through debridement of the root canals and three dimensional seal of the obturated materials, a good preoperative radiographic evaluation is necessary. The anatomy of root canal systems dictates the condition under which root canal therapy is carried out and can directly affect its prognosis. Extra root or root canals if not detected are a major reason for failure of this treatment. Incomplete removal of all the irritants from pulp space may increase the possibility of treatment failure.

This case illustrates some of the difficulties that may arise during root canal treatment. The main reason for unfavourable outcomes in endodontic treatment of mandibular incisors is the inability to detect the presence of a second canal, which then will not be disinfected or filled during treatment. 
It is essential that clinicians know the clinical and radiographic signs that suggest the presence of extra canals. Clinically, the presence of continous bleeding in teeth with pulpitis or normal pulp despite complete instrumentation can suggest the presence of such canals. In case with necrotic pulps or when the canals are pulpless, the presence of an apical rarefaction on the lateral side of the root may suggest the presence of an extra canal. Some of the other indications could be the eccentric location of an endodontic file on a radiograph during working length determination, inconsistent apex locator readings, a sinus tract that traces laterally away from the main canal, or the feeling of a 'catch' on the canal wall during instrumentation of a wide and unobstructed main canal.

Careful evaluation of two or more periapical radiographs is mandatory. These angled radiographs provide much needed information about root canal morphology. Nattress et $\mathrm{al}^{6}$ radiographed 790 extracted mandibular incisors and premolars in order to assess the incidence of canal bifurcation in a root. Using the 'fast break' guideline that the disappearance or narrowing of a canal infers that it divides resulted in failure to diagnose one-third of these divisions from a single radiographic view. The evaluation of the root canal system is most accurate when the dentist uses the information from multiple radiographic views together with a through clinical exploration of the interior and exterior of the tooth.

An important aid for locating root canals is the dental-operating microscope which was introduced into endodontics to provide enhanced lightning and visibility. It brings minute details into clear view. It enhances the dentist's ability to selectively remove dentin with great precision therby minimizing procedural errors. Several studies have shown that it significantly increases the dentist's ability to locate and negotiate canals.

Conventional and modified canal staining and clearing techniques are considered the gold standard method of studying root canal morphology. The CBCT has been also used as a tool to examine root canal morphology in different populations. Neelakantan et $\mathrm{al}^{9}$ demonstrated that $\mathrm{CBCT}$ is a accurate as the canal staining and clearing technique in identifying root canal morphology. It provides a noninvasive and clinically applicable examination of root canal morphology. it is also well established that because of the two-dimensional nature of conventional periapical radiographic films, inevitable geometric distortion, and anatomical noise mean it is not possible to examine the morphology of the root canal morphology of the root canal accurately. Thus, in this study we used CBCT to reconfirm the root canal morphology of mandibular incisors. ${ }^{8}$

Careful examinations of the tooth before and during access cavity preparation are essential along with thorough probing of the pulp chamber. A common reason for not locating a second canal in mandibular incisors is an inadequate access opening into the tooth which leaves a lingual shelf of dentine over the second (usually the lingual canal). Some authors recommended that when entering mandibular incisors in order to perform endodontic treatment, clinicians should always initially assume that two root canals exist. Therefore, clinicians should always prepare an access cavity with the appropriate size and location, and then thoroughly search for the two canals. Only after such a search fails to reveal a second canal should clinicians be satisfied that the tooth has only one root canal.

\section{Conclusion:-}

These case reports highlight the importance of having a through knowledge of all possible root canals irregularities. In some cases, it is very difficult to identify additional root canals by radiographic examinations and therefore visualization and deep probing during initial endodontic treatment is essential for the location of all canals. With advances in modern endodontic techniques, most teeth with complex root canal anatomies can be succesfully treated without surgical intervention.

\section{References:-}

1. Vandana B. Kokane, Swapnil N. Patil, Mohit K. Gunwal, Rajesh Kubde, and Swaraj Atre. Treatment of Two Canals in All Mandibular Incisor Teeth in the Same Patient-Case Report. Case Reports in Dentistry, Hind Publi Corp Volume 2014.

2. Vibha hegde, Sharad r. Kokate Yogesh r. Sahu. An unusual presentation of all the 4 mandibular incisors having 2 root canals in a single patient - A case report. ENDODONTOLOGY.

3. Khalid S. Al-Fouzan, Abdulatif AlManee, Jilan Jan, Mansour Al-Rejaie. Incidence of two canals in extracted mandibularincisors teeth of Saudi Arabian samples. Saudi Endodontic Journal • May-Aug 2012; 2(2)

4. Tiku AM, Kalaskar RR, Damle SG. An unusual presentation of all the mandibular anterior teeth with two root canals - A case report. J Indian Soc Pedo Prev Dent 2005;23:204-6. 
5. Yury S. Kabak, PhD and Paul V. Abbott. Endodontic treatment of mandibular incisors with two root canals: Report of two cases. Aust Endod J 2007; 33: 27-3.

6. Nattress BR, Martin DM. Predictability of radiographic diagnosis of variations in root canal anatomy in mandibularincisor and premolar teeth. Int Endod J 1991;24:58-62.

7. Schwarze T, Baethge C, Stecher T, Geurtsen W. Identification of second canals in the mesiobuccal root of maxillary first and second molars using magnifying loupes or an operating microscope. Aust Endod J 2002; 28:57-60.

8. Hakan Arslan ,Ertas .H, Elif T,Kalabalık F, Ismail D.C. Evaluating root canal configuration of mandibular incisors with cone-beam computed tomography in a Turkish population. Journal of Dental Sciences (2015) 10, 359-364.

9. Neelakantan P, Subbarao C, Ahuja R, Subbarao CV, Gutmann JL. Cone-beam computed tomography study of root and canal morphology of maxillary first and second molars in an Indian population. J Endod 2010;36:6227.

10. Funato A, Funato H, Matsumoto K. Mandibular central incisor with two root canals. Endod Dent Traumatol 1998; 14: 285-286.

11. 11.Vertucci FJ. Root canal anatomy of the mandibular anterior teeth. Journal of American Dental Association 1974;1:89(2):369-71 